\title{
EFECTO DE LA POSICIÓN DE LA YEMA Y DE LA PODA EN PLANTAS DE AGUACATE DESTINADAS A LA CLONACIÓN
}

\author{
EFFECT OF BUD POSITION AND PRUNING ON AVOCADO STOCK PLANTS \\ INTENDED FOR CLONING
}

\author{
Nicole Darrouy Palacios*, Mónica Castro Valdebenito, Ricardo Cautín Morales, Loretto Kort Silva \\ y Rodrigo Bozzolo Artaza
}

Facultad de Agronomía, Pontificia Universidad Católica de Valparaíso, Casilla 4-D, Quillota-Chile. Tel: (56)-32-2274529 y Fax: (56)-32-2274570.

* Autor para correspondencia (paltos@ucv.cl)

\section{RESUMEN}

La técnica de etiolación y acodo es actualmente la principal alternativa de propagación vegetativa implementada a nivel mundial para la multiplicación comercial de clones de aguacate (Persea americana Mill.), cuya etapa de enraizamiento es considerada como la más crítica del proceso y requiere ser optimizada. En este estudio se evaluó el efecto de la posición de la yema dentro del brote a utilizar en el proceso de clonación y del manejo de poda en árboles de aguacate cv. 'Hass' y del portainjerto clonal cv. 'Duke 7', en dos etapas de esta técnica: injertación y crecimiento del brote etiolado. Los resultados mostraron que los injertos procedentes de yemas basales del brote originaron brotes etiolados de mayor grosor $(P \leq$ 0.05) que los ubicados en yemas apicales e intermedias. Los brotes etiolados del portainjerto 'Duke 7' obtenidos de yemas procedentes de las secciones media y basal, tardaron menos tiempo en el periodo de la cámara de etiolación y enraizamiento, que los brotes procedentes de yemas apicales. Los análisis histológicos realizados en brotes etiolados de 'Hass', mostraron un posible efecto de la posición de la yema y del manejo de poda a nivel de plantas madre sobre aspectos anatómicos, como en la continuidad de las fibras de esclerénquima y grosor del cambium fascicular e interfascicular. Finalmente, las púas procedentes de secciones apicales presentaron un mayor contenido de carbohidratos solubles totales que las secciones medias y basales.

Palabras clave: Persea americana, etiolación, injertación, portainjertos clonales, propagación clonal.

\section{SUMMARY}

Etiolation and layering techniques currently represent the main alternative implemented for plant propagation in the world of commercial multiplication of avocado clones (Persea americana Mill.). Rooting stage is considered most critical and needs to be optimized. In this study, an assessment of the effect of bud position inside the shoot to be used during the cloning process was carried out, along with an evaluation of the pruning management in 'Hass' avocado trees and the 'Duke 7' clonal root stocks, in two stages: grafting and growth of the etiolated sprout. Results showed that grafts from the basal buds inside the sprout originated thicker etiolated sprouts $(\mathrm{P} \leq \mathbf{0 . 0 5})$ than those located in the apical or middle buds. The shortest time for obtaining rooted etiolated sprouts in
'Duke 7' rootstocks occurred on buds located in the middle and basal sections, than on sprouts from buds at the apical section. Histological analysis performed on etiolated sprouts of 'Hass' indicated an effect from bud position and pruning management of stock plants on anatomical aspects like in the continuity of sclerenchyma fibers and thickness of the fascicular and interfascicular cambium. Finally, scions from apical sections had higher total soluble carbohydrate content than middle and basal sections.

Index words: Persea americana, etiolation, clonal rootstocks, grafting, clonal propagation.

\section{INTRODUCCIÓN}

En Chile, la mayoría de las plantaciones comerciales de aguacate (Persea americana Mill.) están injertadas en portainjertos originados por semilla. Según Castro et al. (2003), la utilización de este tipo de portainjertos induce niveles de productividad calificados como subóptimos, lo cual puede ser explicado por la heterogeneidad productiva que se genera entre individuos. Esta situación fue poco relevante para la industria hasta hace pocos años debido a que las condiciones de mercado eran estables y promisorias, y las plantaciones se establecían en suelos de buena calidad. Sin embargo, en la actualidad las condiciones de mercado y de cultivo han cambiado; la mayor competitividad actual en la industria y el desplazamiento del cultivo a zonas con limitantes edáficas hace que se requiera del uso de portainjertos clonales con características específicas. Para mejorar los rendimientos y la calidad del fruto, los productores de aguacate deberán utilizar plantas comerciales de excelencia.

Leakey (2004) define la propagación vegetativa como un proceso que puede ser natural o artificial, y este último constituye una poderosa herramienta para el ámbito agrícola, hortícola y forestal en la multiplicación de 
genotipos individuales para la producción de cultivares y clones. Whiley et al. (2007) describen que a fines de los años 70's se desarrolló y patentó en California, Estados Unidos, un sistema de propagación clonal de plantas de aguacate que utiliza la técnica de etiolación y acodo, cuyos avances científicos han estado enfocados principalmente a la optimización del enraizamiento, a través del uso y evaluación de reguladores del crecimiento de tipo auxínico (Brokaw, 1987; Hofshi, 1996; Ernst, 1999; Whiley y Anderson, 2002; Whiley et al., 2007).

Sin embargo, McKenzie et al. (1988) han descrito pérdidas importantes de plantas en viveros, con especial referencia al cv. 'Hass', durante la injertación de la variedad comercial en el portainjerto clonal; los principales factores involucrados en estas pérdidas se han atribuido a: problemas de compatibilidad entre las partes, condiciones ambientales inadecuadas durante la injertación, y al estado fisiológico del portainjerto y del injerto. Esta situación plantea la necesidad de estudiar nuevos factores que también inciden en el éxito de esta técnica.

Baldini (1992), Mesén et al. (2001) y Leakey (2004) destacan la relevancia del manejo de las plantas madre en optimizar la capacidad de enraizamiento de estacas, en cuanto a poda, fertilización, balance en el contenido de carbohidratos y manejo de luz, para obtener brotes de calidad morfológica y fisiológica. McKenzie et al. (1988) afirman que el manejo adecuado de las plantas madre de aguacate permite disminuir la pérdida de plantas durante la injertación. En árboles madre podados severamente, la injertación es más efectiva durante todo el año que en árboles con madera adulta que puede estar produciendo frutos, en los que las pérdidas por injertación estarían asociadas a una menor disponibilidad de reservas en la planta. Es importante entonces considerar que los carbohidratos son los principales compuestos de almacenamiento de reservas en plantas perennes (Castillo et al., 1996). La importancia del estado nutricional de los órganos vegetales destinados a la propagación, fue descrita por Baldini (1992), con especial referencia al papel de los carbohidratos.

Por ello el estudio de estos factores es de importancia para el avance y consolidación de la técnica de propagación clonal de plantas de aguacate en Chile. El objetivo del presente estudio fue evaluar el efecto de la posición de la yema en el brote y de la poda de las plantas madre, en diferentes etapas de la propagación clonal en aguacate.

\section{MATERIALES Y MÉTODOS}

Esta investigación se llevó a cabo en la provincia de Quillota, Región de Valparaíso, Chile, sitio ubicado en las coordenadas geográficas $32^{\circ} 53^{\prime} 44.41^{\prime}$ ' LS y $71^{\circ} 12^{\prime}$ 25.05' LO, a una altitud de $130 \mathrm{~m}$.

\section{Primer ensayo. Efecto de la posición de la yema en el brote y de la poda en árboles madre de aguacate cv. \\ 'Hass', en dos etapas, injertación y desarrollo del brote etiolado, en invernadero sin control de temperatura}

El ensayo se llevó acabo de agosto 2005 a agosto 2006, en un invernadero metálico cubierto con $168 \mathrm{~m}^{2} \mathrm{de}$ polietileno de dos temporadas, de $0.15 \mathrm{~mm}$ de espesor. Este invernadero no tenía sistema de regulación de la temperatura ambiental (invernadero 'frío'), cuyas temperaturas promedio registradas variaron en el rango de 15 a $25^{\circ} \mathrm{C}$. El material vegetal para la injertación fue obtenido de dos árboles de aguacate cv. 'Hass' procedentes del huerto comercial de la Estación Experimental 'La Palma' (32 53' 38.69', LS y $71^{\circ} 12$ ' $16.78^{\circ}$ ' LO; altitud de $130 \mathrm{~m}$ ). Los árboles tenían una edad de siete años: uno de ellos fue severamente podado en el invierno del año anterior, y el otro se encontraba en plena producción (sin poda). Como plantas nodrizas se utilizaron plántulas del cv. 'Ettinger', las cuales fueron sembradas en octubre 2005; cuando sus tallos alcanzaron un diámetro de $5 \mathrm{~mm}$ a $5 \mathrm{~cm}$ del suelo, fueron injertadas mediante injertos de púa con una o dos yemas del tipo cuña o hendidura terminal. En febrero 2006 se recolectaron e injertaron de manera simultánea 60 púas de cada árbol, 30 de la sección apical y 30 de la sección basal. Una vez expandidas las primeras hojas del injerto, cada planta fue trasladada a una cámara de oscuridad ubicada en el laboratorio, la que se mantuvo entre 24 a $30{ }^{\circ} \mathrm{C}$, para promover el crecimiento etiolado de los brotes.

Los tratamientos de "poda" en brotes de plantas madres fueron los siguientes: $\mathrm{T} 1$, brotes provenientes de la sección apical de árboles podados; $\mathrm{T} 2$, brotes provenientes de la sección basal de árboles podados; T3, brotes provenientes de la sección apical de árboles sin poda; T4, brotes provenientes de la sección basal de árboles sin poda. En total fueron cuatro tratamientos, con 30 repeticiones cada uno. El diseño experimental utilizado fue uno completamente al azar unifactorial, donde la unidad experimental estuvo constituida por una planta. Los datos fueron analizados mediante un análisis de varianza y las diferencias significativas entre tratamientos fueron determinadas mediante análisis de comparación de medias con la prueba de Tukey $(\mathrm{P} \leq 0.05)$. 
Las variables medidas fueron: 1) Días transcurridos entre la injertación y expansión de las primeras hojas de las yemas ( 1 a $2 \mathrm{~cm}$ de longitud); y 2) Diámetro del brote etiolado a $10 \mathrm{~cm}$ desde su base al ápice, después de seis semanas en oscuridad. El diámetro se midió con un vernier digital Mitutoyo ${ }^{\circledR}$ (código 500-144, serie 0001325, Japón). Adicionalmente, se extrajeron dos trozos de brotes etiolados procedentes de injertos de las secciones apicales y basales de los árboles de 'Hass' con y sin poda. Para el análisis histológico estos trozos fueron fijados en una solución de formalina, ácido acético y alcohol (10: 5: 50, v/v) (Ruzin, 1999). Luego los trozos fueron deshidratados en una batería de alcoholes de graduación creciente de $70 \%$ a $100 \%$ (v/v), y luego pasados a tres baños de xilol, y finalmente se sumergieron en parafina a punto de fusión $\left(60{ }^{\circ} \mathrm{C}\right)$. Se cortaron secciones entre 12 a $14 \mu \mathrm{m}$ de grosor del tallo incluido en cera, con un micrótomo rotatorio Spencer 820® (American Optical Co., Buffalo, NY, USA). Las secciones se tiñeron con safranina $1 \%$ y verde luz $0.2 \%$. Los cortes fueron observados a $10 \mathrm{X}$ y $40 \mathrm{X}$ con un microscopio óptico Leite ${ }^{\circledR}$, modelo Laborlux $\mathrm{K}$, de origen alemán, conectado a una cámara fotográfica semiautomática.

\section{Segundo ensayo. Efecto de la posición de la yema en el brote y de la poda de árboles madre del portainjerto clonal de aguacate cv. 'Duke 7', en dos etapas, injertación y desarrollo del brote etiolado, en invernadero climatizado}

El segundo ensayo fue realizado entre marzo 2007 y marzo 2008, en un invernadero metálico de $72 \mathrm{~m}^{2}$ cubierto con doble capa de polietileno de dos temporadas, de $0.15 \mathrm{~mm}$ de espesor, con temperatura mantenida entre los 28 a $31^{\circ} \mathrm{C}$ durante todo el día mediante un calefactor de gas propano Tecnoclima ${ }^{\circledR}$ (modelo PA, Italia). El material vegetal utilizado fue obtenido de dos localidades (grupos).

El primer grupo lo constituyeron tres árboles madre injertados con el cv. 'Duke 7' seleccionados por su homogeneidad, que se encontraban sobre el portainjerto franco cv. 'Mexicola' y que fueron establecidos en 2004 en el huerto comercial de la empresa Agroindustrial Pedegua S.A. (32 ${ }^{\circ} 24^{\prime} 39.34^{\prime \prime}$ LS y $71^{\circ} 06^{\prime} 46.04^{\prime \prime}$ LO, a una altitud de $520 \mathrm{~m}$ ), en la provincia de Petorca, Región de Valparaíso. Los árboles madre de 'Duke 7' se encontraban sobre camellones con orientación norte-sur y con una distancia de plantación de $2.5 \times 4 \mathrm{~m}$. Los árboles existentes en esta parcela no habían recibido poda desde el momento de su plantación hasta la fecha de realización del ensayo.
El segundo grupo fue la selección de otros tres árboles madre injertados con 'Duke 7' también injertado sobre el portainjerto 'Mexicola', que fueron establecidos en 2005 en un invernadero no climatizado en el Vivero Limache

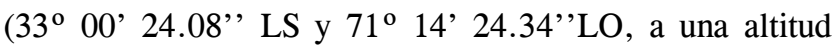
de $134 \mathrm{~m}$ ), provincia de Quillota, Región de Valparaíso. Los árboles en el invernadero se encontraban también sobre camellones, con una orientación norte-sur y con una distancia de plantación de 2 x $1 \mathrm{~m}$, y habían sido podados en noviembre 2006. La poda consistió básicamente en una rebaja de la altura de los árboles y eliminación de brotes y ramas laterales, para mantener un solo eje con ramas principales severamente rebajadas a una o dos yemas. Se utilizaron plántulas nodrizas del cv. 'Esther', las cuales fueron sembradas en julio 2007. El tipo de injerto aquí utilizado fue de cuña o hendidura y comenzó a efectuarse el 4 de diciembre 2007, con púas que fueron extraídas con 10 d de diferencia entre cada sección del brote; este criterio fue aplicado en todos los árboles de 'Duke 7'.

El diseño experimental utilizado fue uno completamente al azar unifactorial, con seis tratamientos: T1) Brotes provenientes de la sección apical de árboles sin poda; T2) Brotes provenientes de la sección media de árboles sin poda; T3) Brotes provenientes de la sección basal de árboles sin poda; T4) Brotes de árboles con poda, de la sección apical; T5) Brotes de árboles con poda, de la sección media; y T6) Brotes de árboles con poda, y de la sección basal. La unidad experimental fue una planta, con 30 repeticiones para cada tratamiento. Los datos fueron analizados mediante un análisis de varianza y la comparación de medias se hizo con la Prueba de Duncan $(\mathrm{P} \leq 0.05)$.

Se midió el tiempo (días) que las plantas demoraron en alcanzar las siguientes etapas de la técnica de etiolación y acodo: 1) Injertación a prendimiento; 2) Prendimiento a ingreso a la cámara de etiolación; y 3) Ingreso a la cámara de etiolación a enraizamiento. Se evaluó el diámetro del brote etiolado a $10 \mathrm{~cm}$ desde la base del brote hacia el ápice, cuando alcanzaron $30 \mathrm{~cm}$ de altura, lo que ocurrió entre 5 y 6 semanas de permanencia en la cámara de etiolación. También se cuantificaron los carbohidratos totales solubles contenidos en púas con yemas provenientes de las diferentes secciones del brote para los árboles de 'Duke 7' con y sin poda, obtenidas simultáneamente y con el mismo criterio de las púas para injertación. La cuantificación se realizó el mismo día de la recolección de la púa, mediante el método de la antrona (Yemm y Willis, 1954), y se utilizaron nueve réplicas por tratamiento. 


\section{RESULTADOS Y DISCUSIÓN}

Primer ensayo. Efecto de la posición de la yema en el brote y de la poda de árboles madre de aguacate cv.

'Hass', en dos etapas, injertación y desarrollo del brote etiolado, en invernadero sin control de temperatura

El análisis de varianza evidenció que no hubo efecto ( $\mathrm{P}$ $\geq 0.05$ ) de los tratamientos aplicados sobre la tasa de desarrollo de los injertos del cv. 'Hass'. Estos resultados contrastan en parte con los de Hartmann et al. (1996) y Whiley et al. (2007), quienes describieron que la mejor madera para la extracción de púas se encuentra en árboles jóvenes o adultos podados para forzar la aparición de rebrotes. Según Thorp y Sedgley (1992), el crecimiento del aguacate está genéticamente determinado y existe una amplia variabilidad entre cultivares; así, 'Gwen' y 'Reed' presentarían una débil dominancia apical y producirían muchos brotes silépticos con una acrotonía débil, mientras que 'Hass' presentaría una situación intermedia en relación con la dominancia apical, con el desarrollo de brotes silépticos y con la acrotonía. Estos antecedentes podrían explicar la homogeneidad que mostraron los tratamientos aquí aplicados en el tiempo que demoraron en desarrollar los injertos en 'Hass'.

En el diámetro de los brotes etiolados (Cuadro 1) hubo diferencias significativas $(\mathrm{P}<0.05)$, ya que el material obtenido de secciones basales originó los brotes de mayor diámetro, sin importar si el árbol había sido podado o no. $\mathrm{Al}$ estudiar la propagación de plantas leñosas por estaquillado, Hartmann et al. (1996) reportaron que hay diferencias en la composición química a lo largo de una estaca, y que la capacidad de enraizamiento de las porciones basales es mayor que las provenientes de partes apicales, lo cual podría estar relacionado con diferencias en la acumulación de carbohidratos. Los mismos autores destacaron que en análisis químicos realizados en ramas de rosal (Rosa spp.), las secciones basales fueron las que presentaron mejor equilibrio en el cociente carbono/nitrógeno, que fue cercano a uno.

Cuadro 1. Diámetro de brotes etiolados de aguacate cv. 'Hass' medidos a $10 \mathrm{~cm}$ desde su base al ápice y desarrollados en cámara de etiolación con temperaturas entre 24 a $30{ }^{\circ} \mathrm{C}$ durante cinco semanas.

\begin{tabular}{lc}
\hline Tratamiento & Diámetro $(\mathrm{mm})$ \\
\hline T1 (Brote de árboles con poda, sección apical) & $3.71 \mathrm{~b}$ \\
T2 (Brote de árboles con poda, sección basal) & $4.10 \mathrm{a}^{\dagger}$ \\
T3 (Brote de árboles sin poda, sección apical) & $3.76 \mathrm{~b}$ \\
T4 (Brote de árboles sin poda, sección basal) & $4.11 \mathrm{a}$ \\
\hline
\end{tabular}

${ }^{\top}$ Medias con letras iguales no son estadísticamente diferentes (Tukey, $0.05)$.
Leakey (2004) describió la existencia de un gradiente de variación en edad del tejido a lo largo del brote, que afectaría el tamaño de las hojas, la longitud y diámetro de los entrenudos, el grado de lignificación del tallo, así como el contenido de nutrientes y carbohidratos, por mencionar algunos. Esta situación explica en parte el hecho de que en la propagación por estaquillado, no existan dos estacas fisiológicamente idénticas en sus respuestas, por ejemplo en formación de raíces advénticias. Según Thorp y Sedgley (1992), el aguacate 'Hass' presenta una dominancia apical y acrotonía intermedia, por lo que el contenido de citocininas sería creciente en el sentido basípeto del brote, situación que podría explicar el mayor vigor de los brotes etiolados provenientes de las secciones basales. Los resultados aquí obtenidos permiten proponer a estas secciones como las más adecuadas para la clonación, atribuible a un adecuado equilibrio carbono/nitrógeno y un mayor contenido de citocininas que darían origen a brotes más vigorosos.

Los análisis histológicos mostraron posibles diferencias en la organización anatómica de los tejidos por efecto de la poda y de la posición de la yema dentro del brote. Los tejidos que componen el tallo de un brote etiolado de aguacate se describen en la Figura 1. Destaca la menor continuidad del anillo esclerenquimatoso en las secciones basales, con respecto a las apicales (Figuras 2 y 3), situación aún más notoria en el material procedente de árboles podados. Flores-Vindas (1999) describió a las fibras del esclerénquima como estructuras celulares presentes en tallos, hojas, raíces y frutos, asociadas con el tejido vascular, sobre todo con el xilema.

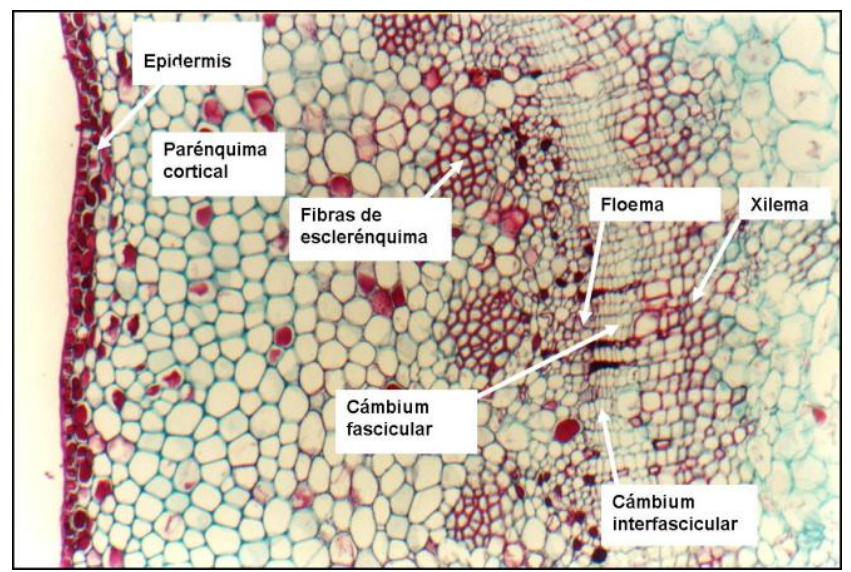

Figura 1. Corte histológico transversal de un brote etiolado de tallo de aguacate cv. 'Hass' (40X). 


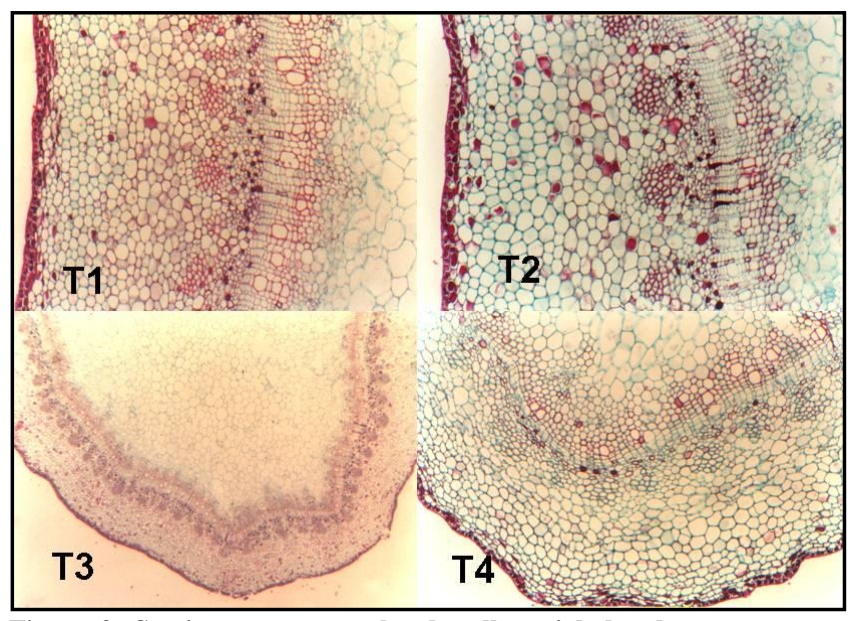

Figura 2. Secciones transversales de tallos etiolados de aguacate cv. 'Hass' (10X). T1: sección apical de árbol con poda; T2: sección basal de árbol con poda; T3: sección apical de árbol sin poda; T4: sección basal de árbol sin poda.

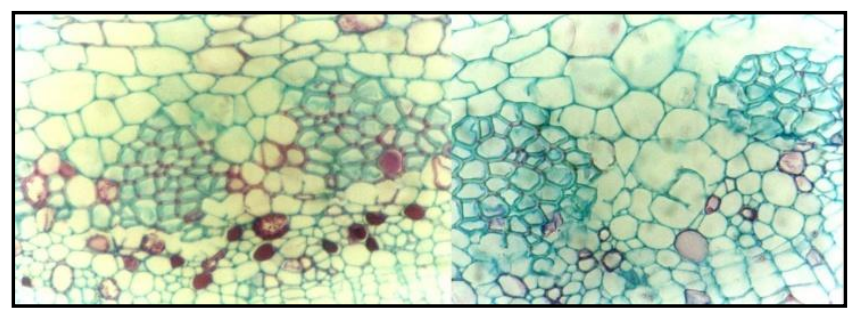

Figura 3. Diferencias en la cercanía de los anillos de esclerénquima, en cortes transversales de brotes etiolados de árboles de aguacate cv. 'Hass' podados. La sección apical de la izquierda presenta mayor continuidad entre los anillos que la sección basal ubicada a la derecha (40X).

Según Baldini (1992), la aplicación de ciertos tipos de preacondicionamiento, como la etiolación de tejidos, produce desorganización del esclerénquima, lo que favorece la formación de raíces adventicias en estacas de tallo de olivo (Olea europaea L.). Si bien, los cortes histológicos fueron realizados a un pequeño número de réplicas muestra y analizados sólo a un nivel descriptivo, los resultados obtenidos en este estudio constituyen una referencia inicial y única en relación a esta temática. $\mathrm{Al}$ respecto, secciones basales de brotes provenientes de árboles de aguacate con y sin poda en los Tratamientos 2 y 4 (Figura 2), manifestaron un aumento en el grosor del cámbium interfascicular y fascicular, que mostraría un posible efecto de la poda y de la posición de la yema dentro del brote. La actividad del cámbium vascular permite que el árbol aumente anualmente su diámetro, al agregar capas sucesivas de floema y xilema secundarios (Flores-Vindas, 1999). De acuerdo con Baldini (1992), la activación del cámbium está directamente ligada con la reactivación de las yemas, la cual ocurre en sentido basípeto en la ramilla y es controlada por la interacción de reguladores de crecimiento como auxinas, citocininas y giberelinas.
Si la relación de auxinas/citocininas cambia con la posición de la yema dentro de la ramilla, las secciones basales tendrían mayor contenido de citocininas y presentarían una mayor actividad mitótica del tejido cambial, lo que podría explicar el mayor grosor de los brotes etiolados. En los Tratamientos 2 y 4 este fenómeno estaría potenciado por la poda del material vegetal que induciría el rejuvenicimiento de sus tejidos. Además, una mayor cantidad de células cambiales en estos tejidos sería una condición beneficiosa para el proceso de injertación ya que participan en la unión y la regeneración de los tejidos vasculares (Hartmann et al. 1996).

Segundo ensayo. Efecto de la posición de la yema en el brote y de la poda de árboles madre del portainjerto clonal de aguacate cv. 'Duke 7', en dos etapas, injertación y desarrollo del brote etiolado, en invernadero climatizado

El material vegetal de los Tratamientos 1 y 4, que corresponden a brotes sin y con poda, provenientes de la sección apical de arboles, respectivamente, demoraron más tiempo en lograr la altura necesaria $(30 \mathrm{~cm})$ para iniciar el proceso de enraizamiento (Cuadro 2). Sin embargo, ninguno de los seis tratamientos tuvieron efecto significativo en las dos etapas anteriores del proceso de clonación, lo que se contrapone a lo planteado por McKenzie et al. (1988) y Whiley et al. (2007), quienes destacaron la importancia de utilizar árboles podados para la obtención de material vegetal adecuado para la propagación. Una posible explicación de tal contraste podría ser el hecho de que los árboles utilizados en este ensayo eran jóvenes, 4 años los no podados y de 3 años los podados.

La demora de las púas de 'Duke 7' procedentes de los Tratamientos $1 \mathrm{y} \mathrm{4}$, pudo deberse en parte a sus menores diámetros y al menor grado de lignificación que presentaban al momento de la extracción de la planta madre, en comparación con las secciones medias y basales. Al respecto, McKenzie et al. (1988) consideran que una de las principales causas de muerte de injertos en el cv. 'Hass' es la combinación de altas temperaturas ambientales al momento de la injertación con el uso de púas inmaduras, poco lignificadas. Leakey (2004) también describió la importancia del vigor y grado de lignificación de las púas.

El diámetro de los brotes etiolados del portainjerto clonal 'Duke 7' mostró que los Tratamientos 3 y 6, ambos con brotes basales, superaron $(\mathrm{P} \leq 0.05)$ a los demás tratamientos (Cuadro 3), lo que destaca nuevamente la importancia de la posición de la yema dentro del brote en el desarrollo vigoroso de éstos. Estos resultados 
concuerdan con los obtenidos en el Ensayo 1, en cuanto a los diámetros de brotes etiolados y en que las yemas procedentes de secciones basales de árboles con y sin poda lograron diámetros mayores que los provenientes de secciones apicales.

Los contenidos de azúcares solubles totales en púas del portainjerto clonal 'Duke 7' recolectadas durante el mes de diciembre mostraron diferencias significativas entre los tratamientos, donde se pudo evidenciar la relevancia que tendría la posición de la yema dentro del brote en el contenido de estos compuestos de reservas en la madera de la púa (Cuadro 4). Estos resultados podrían parecer opuesto a lo mencionado por Hartmann et al. (1996), quienes señalaron que las secciones basales de los brotes poseen una relación carbono/nitrógeno alta; sin embargo, esta aseveración pudiera estar relacionada con carbohidratos de reserva, como es el caso del almidón y en este ensayo los carbohidratos cuantificados correspondieron a carbohidratos solubles totales. Según Whiley et al. (2007) en aguacate el máximo contenido de almidón en ramas se produce en primavera, justo antes de la floración, y después va disminuyendo para alcanzar sus menores niveles en otoño. Por tanto, a partir de primavera los carbohidratos de reserva podrían ser desdoblados a carbohidratos solubles para su utilización inmediata por los órganos y tejidos en crecimiento vegetativo y reproductivo.

Cuadro 2. Días transcurridos en tres etapas de la técnica de etiolación y acodo aplicada al portainjerto clonal de aguacate cv. 'Duke 7', en invernadero climatizado con temperaturas entre 28 a $31^{\circ} \mathrm{C}$.

\begin{tabular}{|c|c|c|c|}
\hline \multirow[b]{2}{*}{ Tratamientos } & \multicolumn{3}{|c|}{ Periodo (días) } \\
\hline & $\mathrm{I}-\mathrm{P}$ & $P-C E$ & $\mathrm{CE}-\mathrm{TE}$ \\
\hline $\mathrm{T}_{2}$ : Brote de árboles sin poda, sección media & $23.1 \mathrm{a}$ & $9.3 \mathrm{a}$ & $39.6 \mathrm{~b}$ \\
\hline $\mathrm{T}_{4}$ : Brote de árboles con poda, sección apical & $26.8 \mathrm{a}$ & $8.3 \mathrm{a}$ & $46.9 \mathrm{a}$ \\
\hline T5: Brote de árboles con poda, sección media & $20.3 \mathrm{a}$ & $9.7 \mathrm{a}$ & $42.7 \mathrm{~b}$ \\
\hline T6: Brote de árboles con poda, sección basal & $22.4 \mathrm{a}$ & $9.5 \mathrm{a}$ & $33.2 \mathrm{~b}$ \\
\hline
\end{tabular}

I: injertación; P: prendimiento; CE: ingreso cámara de etiolación; TE: tratamiento de enraizamiento. 'Medias con letras iguales en cada columna no son estadísticamente diferentes (Duncan, 0.05).

Cuadro 3. Diámetro de brotes etiolados del portainjerto clonal de aguacate cv. 'Duke 7' medidos a $10 \mathrm{~cm}$ desde su base al ápice y desarrollados en invernadero climatizado con temperaturas entre 28 a $31^{\circ} \mathrm{C}$.

\begin{tabular}{ll}
\hline Tratamientos & Diámetro $(\mathrm{mm})$ \\
\hline $\mathrm{T}_{1}:$ Brote de árboles sin poda, sección apical & $3.4 \mathrm{~b}$ \\
$\mathrm{~T}_{2}$ : Brote de árboles sin poda, sección media & $3.6 \mathrm{~b}$ \\
$\mathrm{~T}_{3}$ : Brote de árboles sin poda, sección basal & $4.2 \mathrm{a}^{\dagger}$ \\
$\mathrm{T}_{4}:$ Brote de árboles con poda, sección apical & $3.7 \mathrm{~b}$ \\
$\mathrm{~T}_{5}$ : Brote de árboles con poda, sección media & $3.4 \mathrm{~b}$ \\
$\mathrm{~T}_{6}$ : Brote de árboles con poda, sección basal & $3.9 \mathrm{a}$ \\
\hline
\end{tabular}

${ }^{\dagger}$ Medias con letras iguales no son estadísticamente diferentes (Duncan, 0.05).

Cuadro 4. Contenido de azúcares solubles totales ( $\mathrm{mg} \mathrm{g}^{-1}$ de p. s.), en púas del portainjerto clonal de aguacate cv. 'Duke 7', según la posición de la púa en la ramilla y el manejo de poda.

\begin{tabular}{lc}
\hline Tratamientos & Carbohidratos $\left(\mathrm{mg} \mathrm{g}^{-1}\right.$ de p. s.) \\
\hline $\mathrm{T}_{1}:$ Brote de árboles sin poda, sección apical & $47.39 \mathrm{a}$ \\
$\mathrm{T}_{2}:$ Brote de árboles sin poda, sección media & $24.39 \mathrm{~b}$ \\
$\mathrm{~T}_{3}:$ Brote de árboles sin poda, sección basal & $17.98 \mathrm{c}$ \\
$\mathrm{T}_{4}:$ Brote de árboles con poda, sección apical & $32.06 \mathrm{a}$ \\
$\mathrm{T}_{5}:$ Brote de árboles con poda, sección media & $30.80 \mathrm{~b}$ \\
$\mathrm{~T}_{6}:$ Brote de árboles con poda, sección basal & $17.14 \mathrm{c}$ \\
\hline
\end{tabular}

Medias con letras iguales no son estadísticamente diferentes (Duncan, 0.05). p. s: peso seco. 
En relación a esto último, la época en que fueron extraídas las púas para la cuantificación de carbohidratos correspondió al inicio del periodo de crecimiento o 'flush' radicular en la zona de realización del ensayo ( $\mathrm{F}$. Hernández G., Com. personal ${ }^{1}$ ). La distribución del carbono dentro de la planta depende de complejas reglas que están relacionadas a los órganos exportadores o "source" que son principalmente los brotes, y los órganos demandantes o "sink" que corresponden a los frutos y raíces (Génard et al., 2008). El contenido de carbohidratos solubles totales cuantificados en púas de 'Duke 7' obtenidas en este ensayo se podría relacionar también con los estudios realizados por Castillo et al. (1996), quienes cuantificaron estos mismos azúcares en brotes e inflorescencias indeterminadas del aguacate 'Colín V-33', y encontraron que el contenido de azúcares solubles totales es mayor en brotes que en inflorescencias, lo que hace suponer que los brotes por su alta actividad metabólica ejercen una mayor demanda de estos compuestos que las estructuras florales.

Así, al tener en consideración la época de obtención del material vegetal (púas), el ciclo fenológico y el hábito de crecimiento del aguacate, éstos regulados por la interacción de la dominancia apical y la acrotonía que controlan la expresión de brotes prolépticos y silépticos, es posible esperar que los brotes apicales ejercerían una mayor demanda de carbohidratos, que los brotes procedentes de secciones medias y basales.

\section{CONCLUSIONES}

La posición de la yema en el brote del aguacate 'Hass' y en el portainjerto clonal 'Duke 7', influyen en el desarrollo posterior de brotes etiolados, puesto que las yemas obtenidas de la sección basal fueron las que originaron los brotes más vigorosos. En el portainjerto 'Duke 7', las yemas de brotes procedentes de las secciones medias y basales demoraron menos tiempo en la etapa de etiolación y enraizamiento, que los brotes procedentes de las secciones apicales. A nivel anatómico, tanto la posición de la yema en el brote como el manejo de poda producen un posible aumento en el desarrollo de cámbium interfascicular y fascicular en brotes etiolados de aguacate 'Hass'. El contenido de carbohidratos solubles, mostró influencia directa de la posición de la yema dentro del brote y de la etapa fenologíca en la época de recolección del material vegetal, ya que las púas de las secciones apicales cosechadas durante el mes de

${ }^{1}$ F Hernández G (1991) Ingeniero Agrónomo. Tesis de Titulación: Aproximación al ciclo fenológico del Palto (Persea americana Mill.), cultivar Hass, para la zona de Quillota, V región. Pontificia Universidad Católica de Valparaíso. Facultad de Agronomía. Quillota, Chile. diciembre presentaron un mayor contenido de carbohidratos (azúcares solubles totales), que las secciones medias y basales.

\section{AGRADECIMIENTOS}

La presente investigación fue realizada en el marco del proyecto de investigación: "Evaluación Agronómica y Propagación de nuevos Portainjertos y Variedades de aguacate en distintas zonas agroclimáticas de Chile", patrocinado por el Fondo de Fomento al Desarrollo Científico y Tecnológico (FONDEF).

\section{BIBLIOGRAFÍA}

Baldini E (1992) Arboricultura General. Ed. Mundi-Prensa. Madrid, España. $379 \mathrm{p}$

Brokaw W H (1987) Avocado clonal rootstock propagation. Proc Internatl. Plant Propag. Soc. 37:97-103.

Castro M, C Fassio, N Darrouy, A Ben-Ya'acov (2003) Determinación de rangos de variabilidad en los niveles de producción del cultivar Hass sobre portainjertos de semilla de raza Mexicana en Chile. In: Actas V Congreso Mundial del Aguacate. Granada- Málaga, España. Octubre 19-24. pp:155160.

Castillo A M, M T Colinas, A Martínez, M L Ortega (1996) Fluctuación anual de carbohidratos en aguacate (Persea americana Mill.) cv. Colín V-33. In: Memorias de la Fundación Salvador Sánchez Colín-CICTAMEX, S.C. Coatepec Harinas, México. pp:45-55. Disponible en: http://www.avocadosource.com/Journals/CICTAMEX/CICTA MEX_1996/ Fitos_96.pdf (Marzo 2008).

Ernst A (1999) Micro cloning: A multiple cloning technique for avocado using micro containers. Rev. Chapingo S. Hort. 5:217220.

Flores-Vindas E (1999) La Planta: Estructura y Función. Vol. II. Ed. Tecnológica de Costa Rica, Costa Rica. 367 p.

Génard M, J Dauzat, N Franck, F Lescourret, P V Moitrier, G Vercambre (2008) Carbon allocation in fruit trees: from theory to modelling. Trees Struct. Funct. 22:269-282.

Hartmann H T, D E Kester, F T Davies, R L Geneve (1996) Plant Propagation: Principles and Practices. 6th ed. Prentice-Hall, Englewood Cliffs, N.J. 770 p.

Hofshi R (1996) Experiments with cloning avocado rootstocks. California Avocado Soc. Yrbk. 80:103-108.

Leakey R R B (2004) Physiology of vegetative reproduction. In: Encyclopedia of Forest Sciences. J Burley, E Evans, J A Younquist (eds). Academic Press. London, UK. pp:1655-1668.

Mckenzie C B, B N Wolstenholme, P Allan (1988) Evaluation of nursery procedures to eliminate graft-take problems. South African Avocado Growers Assoc. Yrbk. 11:48-52. Disponible en: http://www.avocadosource.com/wac2/wac2_p375.htm (Diciembre 2008)

Mesén F, R R B Leakey, C Newton (2001) The influence of stockplant environment on morphology, physiology and rooting of leafy stem cuttings of Albizia guachapele. New For. 22:213-227.

Ruzin S E (1999) Plant Microtechnique and Microscopy. Oxford Univ. Press. New York, USA. 322 p.

Thorp G, M Sedgley (1992) Shoot growth and tree architecture in range of avocado cultivar. In: Proc. 2nd World Avocado Congress. C J Lovatt (ed). Orange CA, USA. April 21-26. University of California-Riverside. pp:237-240. 
Yemm E W, A J Willis (1954) The estimation of carbohydrates in plant extracts by anthrone. Biochem. J. 57:508-514.

Whiley A W, B Schaffer, B N Wolstenholme (2007) El Aguacate: Botánica, Producción y Usos. Puelma, P. CABI, Ediciones Universitarias de Valparaíso. Valparaíso, Chile. 380 p.
Whiley A W, G Anderson (2002) Part Study Tour of South Africa, New Zealand, the UK and the USA (also incorporating Chile). 2: Avocado Nurseries and Rootstock Cloning. 12p. Disponible en: http://www.avocadosource.com/papers/Research_Articles/ WhileyTony2002/WhileyTony2002a.pdf (Noviembre 2008). 